\title{
Rapid weight gain during infancy and subsequent adiposity: a systematic review and meta-analysis of evidence
}

Miaobing Zheng ${ }^{1}$, Karen E Lamb ${ }^{1}$, Carley Grimes ${ }^{1}$, Rachel Laws ${ }^{1}$, Kristy Bolton ${ }^{2}$, Ken K.

$\mathrm{Ong}^{3}$, Karen Campbell ${ }^{1 *}$

${ }^{1}$ Deakin University, Geelong, Australia, Institute for Physical Activity and Nutrition, School of Exercise and Nutrition Sciences

${ }^{2}$ Deakin University, Geelong, Australia, School of Exercise and Nutrition Sciences

${ }^{3}$ Medical Research Council Epidemiology Unit, and Department of Paediatrics, University of Cambridge, Cambridge, UK

Corresponding author

Miaobing Zheng

Institute for Physical Activity and Nutrition, Deakin University

221 Burwood Highway, Burwood, VIC, 3125

$+61392468502$

j.zheng@deakin.edu.au

Word count: 4176

Keywords: Rapid weight gain, infancy, adiposity, overweight risk

Running title: Rapid weight gain during infancy and later adiposity

This is the author manuscript accepted for publication and has undergone full peer review but has not been through the copyediting, typesetting, pagination and proofreading process, which may lead to differences between this version and the Version of Record. Please cite this article as doi: $10.1111 /$ obr.12632

This article is protected by copyright. All rights reserved. 
Acknowledgement: None

Potential conflicts of interest: None

Abbreviations

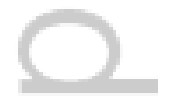

RWG: Rapid weight gain

AGA: Appropriate-for-gestational age

BMI: body mass index

WC: waist circumference

CI: confidence intervals

OR: odds ratio

WHR: waist-to-hip ratio

IOTF: international obesity task force

CDC: centre for disease control

WHO: world health organisation

This article is protected by copyright. All rights reserved. 


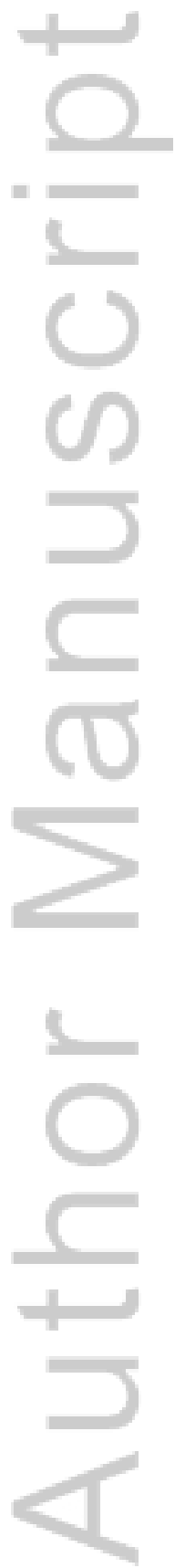

This article is protected by copyright. All rights reserved. 


\section{Abstract (200 max)}

The contribution of rapid weight gain (RWG) during infancy to later adiposity has received considerable investigation. The present systematic review and meta-analysis aimed to update the literature on association between RWG and subsequent adiposity outcomes. Electronic searches were undertaken in EMBASE, MEDLINE, psycINFO, PubMed, and ScienceDirect. Studies that examined the associations between RWG (a change in weight z-scores $>0.67$ ) during infancy (from birth up to age two years) and subsequent adiposity outcomes were included. Random-effects meta-analysis was conducted to obtain the weighted-pooled estimates of the odds of overweight/obesity for those with RWG. Seventeen studies were eligible for inclusion with the majority of studies (15/17) being of high quality and reporting positive associations between RWG during infancy and later adiposity outcomes. RWG in infancy was associated with overweight/obesity from childhood to adulthood (pooled OR = 3.66, $95 \%$ CI: $2.59-5.17, I^{2}>75 \%$ ). Subgroup analyses revealed that RWG during infancy was associated with higher odds of overweight/obesity in childhood than in adulthood; and RWG from birth up to one year was associated with higher odds of overweight/obesity than RWG from birth up to two years. The present study supports that RWG during infancy is a significant predictor of adiposity in later life.

\section{Introduction}

The global estimates indicate an upward trend in childhood obesity over recent decades ${ }^{1}$. In 2015 , over 42 million children globally under the age of five were either overweight or obese 
${ }^{2}$. It is well known that obesity tracks across the life span and childhood obesity is likely to persist into adulthood $^{3,4}$. Further, overweight and obese children are at higher risk of developing many comorbidities later in life ${ }^{4,5}$. Given the high prevalence of childhood obesity and its associated adverse health consequences, tackling childhood obesity has been recognised as a global health priority ${ }^{6,7}$.

The aetiology of obesity is multifactorial and is underpinned by complex interactions of genetic, environmental and psychosocial factors. It has been suggested that infancy is a critical period for development of obesity and its related health outcomes ${ }^{8}$. The contribution of rapid weight gain (RWG) during infancy (up to two years of age) in programming subsequent obesity has sparked much scientific attention ${ }^{8-10}$. The widely accepted definition of RWG is upward centile crossing in weight growth charts. ${ }^{8}$

Two systematic reviews published in 2005 reported consistent positive associations between RWG during infancy and subsequent obesity risk and body mass index (BMI) ${ }^{11,12}$. However, a wide range of effect sizes have been reported due to heterogeneous study designs, including variations in the definition of weight gain during infancy, the length of follow up, the outcomes assessed, the frequency and spread of anthropometric measurements, alongside heterogeneous study populations. A later review conducted by Ong and Loos summarised a total of 21 studies until 2006 and reported standardised results for 15 studies ${ }^{13}$. They concluded that RWG (a change in weight z-score >0.67) during infancy (up to age two years) was associated with a two- to three- fold increase in later overweight/obesity risk in childhood and adulthood, and the effect size of the association was largely influenced by duration of RWG (i.e. from birth to one year or birth to two years), age at outcome 
assessment, and adjustment of confounding factors ${ }^{13}$. For the most part, previous studies in this area have focused on outcomes of obesity risk and BMI. However, since these reviews more relevant studies have emerged. These have examined a broader range of adiposity outcomes across childhood and adulthood ${ }^{10}$. However, no reviews to date have systematically summarised the association between RWG and adiposity outcomes (e.g. general obesity, abdominal and visceral adiposity). The present study aims to provide an update on the previous Ong and Loos review ${ }^{13}$, to look at the association between RWG during infancy and later adiposity outcomes in both childhood and adulthood, and to undertake a meta-analysis to summarise the findings.

\section{Method}

The current review was registered with PROSPERO_registration number CRD42017057698. The conduct and reporting of the present review is based on Preferred Reporting Items for Systematic reviews and Meta-analyses (PRIMSA) checklist ${ }^{14}$.

\section{Eligibility criteria}

The present review included studies reporting an association between RWG during infancy (from birth up to age two years) and subsequent adiposity outcomes. To be eligible, studies needed to include RWG as an exposure with definition of a change in weight-for-age z-score $>0.67$. This is the most frequent and widely accepted definition for RWG ${ }^{12,15}$. The nominated score of 0.67 represents the difference between centile lines on standard growth charts, and an increase of 0.67 can be interpreted as an upward centile crossing through at 
least one centile line ${ }^{13}$. This definition was also chosen to allow later results synthesis in meta-analysis. Further, to be eligible studies must have included at least one type of adiposity outcome measure such as overweight/obesity risk, body mass index (BMI), body fat, waist circumference (WC) or skinfolds measured after the period of RWG. Thus, included studies needed to have multiple adiposity measures taken with a minimum of three time points: birth, one by two years, and one final measure after the second measurement. Studies needed to exclude participants with serious conditions, endocrine or metabolic disorders or severe illness. Conference abstracts without a subsequent publication were excluded from the current review due to insufficient information for data and quality synthesis.

\section{Information source/search}

Electronic searches were conducted in the following electronic databases: EMBASE, MEDLINE, psycINFO, PubMed, and ScienceDirect with the following keywords: infant or early or postnatal; RWG or rapid growth; overweight or obesity or adiposity or body weight or body fat or body mass index or waist circumference or skinfolds. Studies published from March 2006 to January 2017 among human subjects and written in English were included. March 2006 was chosen as the start date because this was the date when the 2006 review ceased their study identification ${ }^{13}$. Manual searches in Google and Google Scholar as well as screening of the reference lists of relevant studies identified from electronic searches were also conducted to identify potential related studies that had not been previously identified.

Study selection and extraction 
Publications identified through searching these five electronic databases were combined and imported into Endnote X7 (Thomson Reuters) with duplicates deleted. In a two stage screening process, two researchers $(\mathrm{MZ}$ and $\mathrm{KB})$ first of all independently screened the titles and abstracts of identified articles. In the second stage, both reviewers independently examined the full texts of articles deemed eligible from title and abstract screening to identify eligible studies for this review. Rationale for study exclusions were documented and excluded studies were grouped based on main exclusion criteria. Inter-reviewer discrepancies were resolved through discussion. Two reviewers (MZ and $\mathrm{KB})$ extracted the following information from included studies: surname of first author, year of publication, study population, duration of RWG, percentage of participants experiencing RWG, type of adiposity outcome and definition, statistical method, adjustment for covariates, and study findings. For studies requiring further information, contacts were made with corresponding authors through emails.

Risk of bias

The quality of included studies was assessed using the Scottish Intercollegiate Guidelines Network 50 (SIGN 50) methodology checklists for cohort studies ${ }^{16}$ and case-control studies ${ }^{17}$. This tool has been recommended as the most appropriate tool to assess the methodological quality of cohort and case-control studies with consideration for selection bias, performance bias, attrition bias, and detection bias ${ }^{18}$. The checklists assess internal validity on five domains: study question, selection of participants, assessment, confounding, and statistical analysis ${ }^{19}$. For cohort studies, recognition of exposure influencing outcome does not apply to our study question and was omitted. An additional two items, derived from 
the National Institute of Health Quality Assessment Tool for Observational Cohort and Cross-Sectional Studies assessing power/sample size/effect size justification and source of funding were added to extend the tool ${ }^{20}$. A total of 15 and 13 items, respectively, was used to assess the quality for cohort study and case-control study. The overall assessment of study quality was rated as high (all and majority of items met with little/no risk of bias), acceptable (most items met with some flaws in study design), and low (most items not met with significant flaws in study design). To minimise bias, quality assessment was conducted by two independent researchers (MZ and $\mathrm{KB})$, and discrepancies between two reviewers were resolved through discussion.

Data synthesis and meta-analysis

Meta-analysis was conducted to examine the pooled estimates of odds ratios (ORs) between RWG during infancy and risk of overweight/obese, as most included studies reported this association. However, conduction of meta-analysis was not possible for other adiposity outcomes due to limited studies. Adjusted odds ratios and corresponding 95\% confidence intervals (CIs) from logistic regression analyses were extracted from the included studies where available. When studies considered RWG during multiple time points in infancy from birth (e.g. birth to one, three, six, nine, 12, 18 and 24 months), the time point closest to two years of age (i.e. from birth to 24 months) was chosen for the analysis to be consistent with our inclusion criteria.

When studies did not present ORs, where possible unadjusted ORs were calculated from reported summary statistics of the numbers of participants who were overweight who did and 
did not have RWG. Adjusted standardized mean differences were converted to ORs according to the formula of Chin et al ${ }^{21}$ when studies considered continuous BMI as the outcome rather than overweight status. The ORs for included studies were transformed to the natural log scale for meta-analysis.

Random effects meta-analysis was conducted using Stata version $14^{22,23}$. Random effects meta-analysis was preferred over fixed effects meta-analysis due to anticipated heterogeneity in the populations considered in the included studies. Forest plots were used to graphically depict the individual and pooled effect sizes. Heterogeneity in effect estimates was assessed using both Chi-squared tests and the $I^{2}$ statistic, the latter of which represents the percentage of variability in the effect estimates due to heterogeneity rather than sampling error. Funnel plots were used to assess potential publication bias.

Subgroup and sensitivity analysis

It was anticipated that the age of participants at outcome assessment would vary between studies, therefore separate subgroup analyses for children ( $<18$ years) and adults $(\geq 18$ years) were conducted. In addition, analyses were stratified by period of RWG, with separate analyses for those considering RWG during the first year of life (specifically from birth to three/four months, to six months, and to one year) and those considering RWG over a longer follow-up period (birth to 18 months, and to 24 months). Finally, in sensitivity analyses, studies deemed to be high risk of bias were omitted from the analysis to assess the influence on research findings. 


\section{Results}

Study selection

The study selection process with reason for exclusion is illustrated in Figure 1. Of 1566 citations, 17 studies met the inclusion criteria that examined RWG from birth up to two years of age using the definition of a change in weight-for-age $\mathrm{z}$-score $>0.67$ and subsequent adiposity outcomes and were included in the present review. For inclusion in meta-analysis on RWG and risk of overweight/obese, two studies reported data from the same cohort ${ }^{24,25}$, and only the study that provided estimates directly relevant to our research question was included ${ }^{24}$. An additional two studies were excluded from the meta-analysis as the relevant data could not be extracted from the data presented and the authors did not provide the required data on request ${ }^{26,27}$. Conversions of estimates were made for two studies not reporting ORs to allow inclusion in the meta-analysis ${ }^{28,29}$, resulting in a final group of 14 studies being included in the meta-analysis.

Study characteristics

Seventeen included studies consisted of twelve prospective cohort studies, four retrospective cohort studies and one case-control study (Table 1). Studies were conducted in eleven countries from five continents: Europe ${ }^{24,25,30,31}$, $\mathrm{US}^{32-34}$, Asia ${ }^{26,35-39}$, Australia ${ }^{28,40}$, Brazil ${ }^{29}$, South Africa ${ }^{27}$ with sample size ranging from 142 to 18,296 participants (Table 1). Of the twelve prospective cohort studies, most studies included healthy term or appropriate-forgestational age (AGA) children with no restrictions on birth weight status, and the subsequent 
adiposity outcomes were measured at ages two to 46.5 years. Retrospective cohort studies included young children aged two/three years ${ }^{34,35}$, and adolescents aged 9-13 years ${ }^{36,38}$. The case-control study included overweight/obese (cases) and normal weight (control) three to five year old children born $>37$ weeks gestation ${ }^{37}$. The duration of RWG examined varied across studies, from birth to three/four months ${ }^{26,35}$, birth to six months ${ }^{29,40}$, birth to one year 27, 28, 31, and birth to 18 months ${ }^{36,38,39}$, and birth to two years ${ }^{30,32,37}$. Two studies examined multiple periods of RWG from birth ${ }^{33,34}$. Twelve out of seventeen studies reported percentage RWG (12.3\% to $54.2 \%)$.

Adiposity outcomes were assessed in early childhood (two years) to mid-adulthood (46.5 years). All studies examined overweight/obesity risk as an outcome, except for two studies ${ }^{27}$, ${ }^{29}$. One study examined the association between RWG and BMI/WC in a small cohort of Brazilian children $(n=167)^{29}$. The other study reported the association between RWG and BMI z, skinfolds, fat mass and fat free mass in a large cohort of South African Children $(n=2352)^{27}$. Three criteria including international obesity task force (IOTF), Centres for Disease Control and Prevention (CDC) growth charts and World Health Organisation (WHO) growth standards were used to categorise participants as overweight/obese. Prevalence of overweight/obesity varied substantially among studies ranging from 5.7\% to $48.5 \%$. Other adiposity outcomes measured included BMI z-score or BMI ${ }^{25,27,29,30}$, waist circumference (WC) ${ }^{29,32,40}$, skinfolds ${ }^{27,30}$, percentage body fat/fat mass ${ }^{27,30,32,39}$, fat free mass ${ }^{27,32}$, waist-to-hip ratio(WHR) ${ }^{40}$, mid upper arm circumference ${ }^{27,30,39}$. Apart from the aforementioned adiposity outcomes, one study also evaluated abdominal and visceral adipose tissue depots ${ }^{32}$. 
Statistical methods used to examine RWG and adiposity outcomes were fairly consistent across studies. Most used multivariable linear and logistic regression models for continuous and categorical outcomes, respectively (Table 1). Other methods utilised were multilevel mixed effect models to adjust for hierarchical nature of the data or test effect of RWG on longitudinal growth trajectories ${ }^{30,39}$, Pearson Chi-square test ${ }^{28}$, and analysis of variance ${ }^{27}$. With respect to adjustment for covariates, large variations were observed between studies. Majority of studies adjusted for both child and maternal factors in their analyses and only one study adjusted for none ${ }^{28}$, and three studies adjusted for child factors only ${ }^{34,35,38}$. Common covariates considered were child sex, birth weight, breastfeeding, maternal body weight status, and maternal education or socio-economic status. Most studies revealed significant association after adjusting for these covariates.

\section{Risk of bias}

Detailed study quality assessment for each item are provided in Supplementary Table 1.The majority of studies (11 of 17) were rated as 'acceptable', with four rated 'high' ${ }^{24-26,39}$ and the remaining two rated 'low' ${ }^{27,28}$. Items pertaining to participation rate, dropout rate and comparison between participants and non-participants were not applicable to retrospective studies. As anthropometric conditions were considered to be pre-existing conditions, the item that whether subjects were free of the outcome at the baseline of the study does not apply in studies that examined anthropometric measures as outcomes (e.g. BMI, WC, skinfolds). 
Assessment of outcome was blinded to exposure in all studies, as researchers would not know who experienced RWG. With the exception of one study ${ }^{30}$, all studies had a clear study question, and most studies recruited or selected subjects from same/similar population at the same time period. Most prospective cohort studies reported a participation rate and dropout rate, and assessed the difference between participants and non-participants. Two studies 27,28 rated as 'low' failed to address most quality assessment items including dropout rate, comparison between participants and non-participants, inadequate adjustment for confounding, lack of reporting on confidence interval and sample size/power justifications.

Study outcomes/results of individual studies

Most studies (15 of 17) reported positive associations between RWG during infancy and later adiposity outcomes. However, among studies that considered different duration of RWG, the within study results were found to vary depending on the period of RWG. For example, Goodell et al found evidence of an association between RWG from birth to one year and odds of overweight/obesity (OR: 11.7, 95\%CI 4.5-30.0), but no evidence of an association when considering RWG from birth to four months (OR $1.9495 \%$ CI $0.89-4.21)^{34}$. Odegaard et al ${ }^{33}$ assessed multiple periods of RWG from birth (birth to one, three, six, nine, 12, 18, 24 months), and only found evidence that RWG from birth to two years was predictive of overweight/obesity at 20-29 years. Two studies found RWG during infancy was not a significant predictor of overweight/obesity at 9-10 years ${ }^{38}$, and overweight/obesity, WC, and WHR at 21 years ${ }^{40}$, respectively. 
Synthesis of results (meta-analysis and sensitivity analysis)

In the meta-analysis combining the ORs for the fourteen eligible studies which considered RWG as a predictor of overweight/obesity (sixteen estimates were combined as two studies presented analyses stratified by sex); RWG during the first two years of life was associated with overweight/obesity in later life from childhood to adulthood (pooled OR $=3.66,95 \%$ CI: 2.59 - 5.17, see Figure 2). Although all estimated ORs were greater than 1, there was substantial heterogeneity in the effect estimates $\left(I^{2}=85.8 \%\right.$, chi-square test $\left.p<0.001\right)$. A funnel plot was assessed to examine the potential for publication bias (Figure 3). Five (31\%) of the sixteen estimates appeared to be outliers. However, there was no clear asymmetry apparent to suggest the presence of publication bias. Results were similar after excluding the single study deemed to be of high risk of bias (pooled OR $=3.58,95 \%$ CI: $2.49-5.14 ; I^{2}=$ $86.6 \%$, chi-square test $p<0.001)$.

One source of heterogeneity was the variation in age at outcome assessment which ranged from 2-3 years in Goodell et al ${ }^{34}$ to 46.5 years in Demerath et al $^{32}$. In subgroup analyses, RWG associations with overweight/obesity in childhood (pooled OR $=4.16,95 \%$ CI: $3.26-$ 5.32; based on 11 studies) were stronger than those with overweight/obesity in adulthood (pooled OR $=2.02,95 \%$ CI: $0.93-4.36$; based on 3 studies). Both analyses showed some heterogeneity in the effect estimates $\left(I^{2}=53.6 \%\right.$, chi-square test $p=0.011$ for childhood; $I^{2}=$ $79.9 \%$, chi-square test $p=0.007$ for adulthood). 
Another source of heterogeneity was the RWG period assessed in infancy, which ranged from birth to three months up to birth to two years. In subgroup analyses, studies which considered RWG up to one year of age had a higher estimated effect size (pooled OR $=4.12,95 \% \mathrm{CI}$ : $1.83-9.28)$ but greater heterogeneity $\left(I^{2}=89.5 \%\right.$, chi-square test $\left.p<0.001\right)$ than those which considered RWG from birth up to two years (pooled OR = 3.58, 95\% CI: $2.67-4.80$; $I^{2}=62.9 \%$, chi-square test $\left.p=0.004\right)$.

\section{Discussion}

Results of the current systematic review and meta-analysis support the findings from previous reviews ${ }^{11-13,41}$. Our findings provide the first quantitative synthesis of systematicallyidentified studies of infant RWG and subsequent overweight/obesity risk. Children experiencing RWG during the first two years of life had 3.66 times (95\%CI 2.59 - 5.17) greater odds of being overweight/obese later in life (from 2 to 46.5 years) than those who did not experience RWG. This is substantially higher than was previously reported in a nonsystematic individual-level meta-analysis of ten cohort studies (OR: 1.97, 95\% CI 1.83, 2.12), and this OR is for an increase $>1$ weight $\mathrm{z}$-score, not 0.67 weight $\mathrm{z}$-score) ${ }^{42}$. Although associations may differ by child sex, this was rarely studied in the included studies. Within our study there is considerable heterogeneity between estimates. Potential sources of heterogeneity were RWG period, prevalence of RWG, age when outcome was measured, covariates adjustment (none, child factors only, both child and maternal factors), and prevalence of overweight/obesity. Subgroup analyses by two potential sources of 
heterogeneity: age at outcome assessment and period of RWG revealed that higher estimates were reported by studies of overweight/obesity in children and studies that considered RWG between birth up to one year of age than studies assessed overweight/obesity in adulthood and RWG between birth up to two years of age. Although no further quantitative synthesis other than overweight/obesity was performed, due to wide variety of adiposity measures used and limited studies that examined each adiposity measures, we found highly consistent associations between RWG and higher measures of adiposity such as percentage body fat, fat mass, abdominal and visceral adipose tissue.

The findings that RWG during infancy predicted higher risk of overweight/obesity in childhood than in adulthood is expected. Unmeasured and residual confounding during the long study period is possible and may contribute to smaller effects seen in those studies with adulthood as the outcome. Moreover, the smaller numbers of studies assessed the obesity risk in adulthood relative to childhood is a plausible contributing factor. The period of RWG had differential effects on later overweight/obesity risk, with studies assessing RWG in first year of life demonstrating a larger estimate highlighting that early infancy is a critical period for development of later obesity. Emerging studies have attempted to evaluate the critical time periods of weight gain in infancy and childhood that are most predictive of later obesity, but have come to mixed findings and the reason why a certain period is more sensitive than others remain unclear ${ }^{43-47}$. This finding is likely contributed by a myriad of factors such as difference in study population, definition and duration of RWG, ages at which anthropometric data were available, accuracy of anthropometrics measurement, duration of follow-up, statistical power and sample size. 
The mechanism through which infant RWG programs subsequent adiposity remains unclear $^{41}$. The association between infant RWG and later adiposity may be influenced by a number of child and maternal factors. It is speculated that the adverse effects of infant RWG on later adiposity is in part contributed by birth weight ${ }^{8}$. RWG is most likely to occur during infancy among low birth weight infants, and intuitively following a period of growth restriction ${ }^{8}$. Infants of low birth weight are more likely to have higher adrenal androgen levels, insulin resistance and central fat deposition, thus heighted vulnerability to weight gain ${ }^{48}$. Reducing the low birth weight incidence through prenatal maternal intervention may be a promising approach to combat obesity risk through lowering the incidence of postnatal RWG. However, the majority of studies in the present review showed that significant associations between RWG and obesity remained after adjusting for child birth weight. Further, studies reporting the positive link were conducted among participants who were born term or AGA and no exclusion was made to low birth weight infants. In other words, greater propensity of rapid growers to obesity may not be confined only to pre-term or small for gestational age children.

Evidence suggests that nutrition in early life has more profound effects on body weight status than other periods in life ${ }^{49}$. A study among AGA term children revealed that the deleterious effect of RWG in infancy on obesity is modified by nutrition in infancy ${ }^{50}$. Among children experienced RWG, those who had been exclusively breastfed for four months had a lower percentage body fat from ages two to five years than did those who had not been exclusively breastfed ${ }^{50}$. Similarly, one study reported that among formula or mixed fed infants, energy intake at four months was associated greater weight gain from birth to age one, two, or three 
years as well as higher BMI at ages one to five years ${ }^{51}$. Interventions designed to reduce infant RWG through early nutrition management may be a more feasible and practical approach for obesity prevention ${ }^{52}$. Maternal factors such as body weight, smoking status,

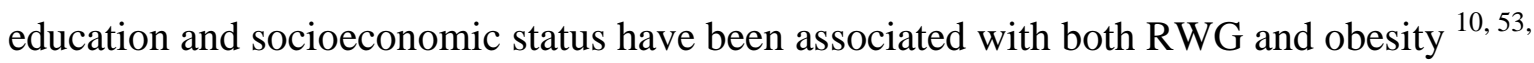
${ }^{54}$. Most studies in the present review considered these important covariates in the analysis, however, further investigations to extricate the effects of these factors on the association are needed ${ }^{41}$

The growing body of studies confirm a positive association between RWG during infancy and higher adiposity measures including WC, skinfolds, fat mass, and abdominal or visceral fat depots. Abdominal obesity as a risk factor of various adverse health outcomes such as cardiovascular and metabolic disease underlies a potential link between RWG in early life and later health risks ${ }^{55}$. Infancy weight gain consists of gain in both fat and lean mass. Some commentaries have questioned whether rapid gains in fat or lean mass during infancy have differential effects on development of later adiposity outcomes and further exploration is required ${ }^{8,56}$

The present review used a systematic approach to summarise the literature on RWG during infancy and later adiposity and meta-analysis was conducted to synthesize effects of RWG on later overweight/obesity risk. Searches were conducted in five electronic databases to identify studies. In comparison to the earlier non-systematic reviews that lacked detailed reporting on data extraction and quality assessment ${ }^{10,13,41}$, the study extraction and quality assessment were performed by two independent reviewers and the influence of study quality on pooled estimates was evaluated in the sensitivity analysis. Low quality studies had minor effects on 
the summary estimates of association between RWG and overweight/obesity risk. The review was limited to publications written in English. Non-English publications relevant to our study question may exist but were not captured in our review. The summary estimates of the metaanalysis may be limited by the considerable heterogeneity among studies. However, we used random-effect meta-analysis that allow heterogeneity among studies and performed subgroup analyses to assess the effects of two major sources of heterogeneity on the association. The effects of other sources of heterogeneity such as covariate adjustment and overweight/obesity prevalence on pooled estimates were not examined due to insufficient information. We attempted to include all studies in the meta-analysis. For studies that did not report ORs, where possible, unadjusted ORs were calculated. We were not able to include two studies in our meta-analysis due to insufficient information. Attempts were made to contact the authors, but no reply was returned. The funnel plot suggests no presence of publication bias. It has to be noted that due to the observational design of studies included in the current review, a causal link between RWG and adiposity cannot be implied, and residual and unmeasured confounding cannot be dismissed.

\section{Conclusions}

In conclusion, our systematic review and meta-analyses found strong associations between RWG during infancy and subsequent overweight/obesity risk. Furthermore, emerging studies reported that RWG during infancy is also associated with various adiposity measures such as body fat and adipose tissue depot. Further studies should explore RWG in development of fat versus lean mass in later life. The current update on the literature underscores the importance of targeting RWG during infancy for obesity prevention. 


\section{References}

1 de Onis M, Blossner M, Borghi E. Global prevalence and trends of overweight and obesity among preschool children. Am J Clin Nutr. 2010; 92: 1257-64.

2 World Health Organization. Report of the commission on ending childhood obesity. WHO Press: Geneva, Switzerland 2016.

3 Serdula MK, Ivery D, Coates RJ, Freedman DS, Williamson DF, Byers T. Do obese children become obese adults? A review of the literature. Prev Med. 1993; 22: 167-77.

4 Herman KM, Craig CL, Gauvin L, Katzmarzyk PT. Tracking of obesity and physical activity from childhood to adulthood: the Physical Activity Longitudinal Study. Int J Pediatr Obes. 2009; 4: 281-8.

5 Guh DP, Zhang W, Bansback N, Amarsi Z, Birmingham CL, Anis AH. The incidence of co-morbidities related to obesity and overweight: a systematic review and meta-analysis. BMC Public Health. 2009; 9: 88.

6 World Health Organsization. Report of the commission on ending childhood obesity. Geneva, Switzerland 2016.

7 Biro FM, Wien M. Childhood obesity and adult morbidities. Am J Clin Nutr. 2010; 91: 1499S-505S.

8 Gillman MW. Early infancy as a critical period for development of obesity and related conditions. Nestle Nutr Workshop Ser Pediatr Program. 2010; 65: 13-20; discussion 20-4.

9 Ellis KJ. Body composition in infancy: impact on health later in life. Nestle Nutr Workshop Ser Pediatr Program. 2010; 65: 213-20; discussion 21-4. 
10 Woo Baidal JA, Locks LM, Cheng ER, Blake-Lamb TL, Perkins ME, Taveras EM. Risk Factors for Childhood Obesity in the First 1,000 Days: A Systematic Review. Am J Prev Med. 2016; 50: 761-79.

11 Baird J, Fisher D, Lucas P, Kleijnen J, Roberts H, Law C. Being big or growing fast: systematic review of size and growth in infancy and later obesity. BMJ (Clinical research ed). 2005; 331: 929 .

12 Monteiro PO, Victora CG. Rapid growth in infancy and childhood and obesity in later life--a systematic review. Obesity reviews : an official journal of the International Association for the Study of Obesity. 2005; 6: 143-54.

13 Ong KK, Loos RJ. Rapid infancy weight gain and subsequent obesity: systematic reviews and hopeful suggestions. Acta Paediatr. 2006; 95: 904-8.

14 Moher D, Shamseer L, Clarke M, Ghersi D, Liberati A, Petticrew M, et al. Preferred reporting items for systematic review and meta-analysis protocols (PRISMA-P) 2015 statement. Syst Rev. 2015; 4: 1.

15 Ong KK, Ahmed ML, Emmett PM, Preece MA, Dunger DB. Association between postnatal catch-up growth and obesity in childhood: prospective cohort study. BMJ (Clinical researched). 2000; 320: 967-71.

16 Scottish Intercollegiate Guidelines Network. Methodology checklist 3: Cohort studies. SIGN 50: A Guideline Developers' Handbook. The Network; 2012: Edinburgh, Scotland 2012.

This article is protected by copyright. All rights reserved. 
17 Scottish Intercollegiate Guidelines Network. Methodology checklist 4: Case-control studies. SIGN 50: A Guideline Developers' Handbook The Network: Edinburgh, Scotland 2012.

18 Bai A, Shukla V, Bak G, Wells G. Quality Assessment Tool Project Report. Canadian Agency for Drugs and Tech-nologies in Health: Ottawa, ON, Canada 2012.

19 West S, King V, Carey T, Lohr K, McKoy N, Sutton S, et al. System to rate the strength of scientific evidence: summary. AHRQ Publication No. 02-E015. Agency for Healthcare Research and Quality: Rockville, MD 2002.

20 National Heart L, and Blood Institute. Study quality assessment tools. 2014.

21 Chinn S. A simple method for converting an odds ratio to effect size for use in metaanalysis. Stat Med. 2000; 19: 3127-31.

22 Harris R, Bradburn M, Deeks J, Harbord R, Altman D, Sterne J. Metan: fixed- and random-effects meta-analysis. The Stata Journal. 2008; 8: 3-28.

23 Sterne J, Harbord R. Funnel plots in meta-analysis. The Stata Journal 2004; 4: 127 41.

24 Heppe DH, Kiefte-de Jong JC, Durmus B, Moll HA, Raat H, Hofman A, et al. Parental, fetal, and infant risk factors for preschool overweight: the Generation R Study. Pediatr Res. 2013; 73: 120-7.

25 Taal HR, Vd Heijden AJ, Steegers EA, Hofman A, Jaddoe VW. Small and large size for gestational age at birth, infant growth, and childhood overweight. Obesity (Silver Spring). $2013 ; 21: 1261-8$.

This article is protected by copyright. All rights reserved. 

infant growth, and childhood body mass index: Hong Kong's children of 1997 birth cohort. Archives of pediatrics \& adolescent medicine. 2008; 162: 212-8.

27 Salgin B, Norris SA, Prentice P, Pettifor JM, Richter LM, Ong KK, et al. Even transient rapid infancy weight gain is associated with higher BMI in young adults and earlier menarche. Int J Obes (Lond). 2015; 39: 939-44.

28 Webster V, Denney-Wilson E, Knight J, Comino E. Describing the growth and rapid weight gain of urban Australian aboriginal infants. J Paediatr Child Health. 2013; 49: 30308.

29 Goncalves FC, Amorim RJ, Eickmann SH, Lira PI, Lima MC. The influence of low birth weight body proportionality and postnatal weight gain on anthropometric measures of 8year-old children: a cohort study in Northeast Brazil. Eur J Clin Nutr. 2014; 68: 876-81.

30 Karaolis-Danckert N, Buyken AE, Bolzenius K, De Faria CP, Lentze MJ, Kroke A. Rapid growth among term children whose birth weight was appropriate for gestational age has a longer lasting effect on body fat percentage than on body mass index. Am J Clin Nutr. 2006; 84: 1449-55.

31 Weng SF, Redsell SA, Nathan D, Swift JA, Yang M, Glazebrook C. Estimating overweight risk in childhood from predictors during infancy. Pediatrics. 2013; 132: e414-21. 32 Demerath EW, Reed D, Choh AC, Soloway L, Lee M, Czerwinski SA, et al. Rapid postnatal weight gain and visceral adiposity in adulthood: The fels longitudinal study. Obesity. 2009; 17: 2060-66. 
33 Odegaard AO, Choh AC, Nahhas RW, Towne B, Czerwinski SA, Demerath EW. Systematic Examination of Infant Size and Growth Metrics as Risk Factors for Overweight in Young Adulthood. PLoS ONE. 2013; 8.

34 Goodell LS, Wakefield DB, Ferris AM. Rapid weight gain during the first year of life predicts obesity in 2-3 year olds from a low-income, minority population. J Community Health. 2009; 34: 370-75.

35 Akaboshi I, Haraguchi Y, Mizumoto Y, Kitano A, Kan H. Taller stature after postnatal rapid weight gain in early infancy predicts overweight status at age 3. Acta Paediatrica. 2008; 97: 1460-64.

36 Fujita Y, Kouda K, Nakamura H, Iki M. Association of rapid weight gain during early childhood with cardiovascular risk factors in Japanese adolescents. J Epidemiol. 2013; 23: 103-08.

37 Rathnayake KM, Satchithananthan A, Mahamithawa S, Jayawardena R. Early life predictors of preschool overweight and obesity: a case-control study in Sri Lanka. BMC public health. 2013; 13: 994.

38 Nanri H, Shirasawa T, Ochiai H, Ohtsu T, Hoshino H, Kokaze A. Rapid weight gain during early childhood is associated with overweight in preadolescence: A longitudinal study in Japan. Child Care Health Dev. 2016; 42: 261-66.

39 Zhou J, Dang S, Zeng L, Gao W, Wang D, Li Q, et al. Rapid infancy weight gain and 7-to 9-year childhood obesity risk a prospective cohort study in rural western China. Medicine. 2016; 95: e3425.

This article is protected by copyright. All rights reserved. 
40 Sutharsan R, O'Callaghan MJ, Williams G, Najman JM, Mamun AA. Rapid growth in early childhood associated with young adult overweight and obesity--evidence from a community based cohort study. J Health Popul Nutr. 2015; 33: 13.

41 Stettler N, Iotova V. Early growth patterns and long-term obesity risk. Current opinion in clinical nutrition and metabolic care. 2010; 13: 294-9.

42 Druet C, Stettler N, Sharp S, Simmons RK, Cooper C, Smith GD, et al. Prediction of childhood obesity by infancy weight gain: an individual-level meta-analysis. Paediatric and perinatal epidemiology. 2012; 26: 19-26.

43 Antonisamy B, Vasan SK, Geethanjali FS, Gowri M, Hepsy YS, Richard J, et al. Weight Gain and Height Growth during Infancy, Childhood, and Adolescence as Predictors of Adult Cardiovascular Risk. J Pediatr. 2017; 180: 53-61.e3.

44 Araujo de Franca GV, De Lucia Rolfe E, Horta BL, Gigante DP, Yudkin JS, Ong KK, et al. Associations of birth weight, linear growth and relative weight gain throughout life with abdominal fat depots in adulthood: the 1982 Pelotas (Brazil) birth cohort study. Int J Obes (Lond). 2016; 40: 14-21.

45 Liem ET, van Buuren S, Sauer PJJ, Jaspers M, Stolk RP, Reijneveld SA. Growth during Infancy and Childhood, and Adiposity at Age 16 Years: Ages 2 to 7 Years Are Pivotal. J Pediatrics. 2013; 162: 287-92.e2.

46 Gonzalez DA, Nazmi A, Victora CG. Growth from birth to adulthood and abdominal obesity in a Brazilian birth cohort. Int J Obes (Lond). 2010; 34: 195-202.

This article is protected by copyright. All rights reserved. 
Terneuzen birth cohort: BMI changes between 2 and 6 years correlate strongest with adult overweight. PLoS One. 2010; 5: e9155.

48 Ong KK, Potau N, Petry CJ, Jones R, Ness AR, Honour JW, et al. Opposing influences of prenatal and postnatal weight gain on adrenarche in normal boys and girls. The Journal of clinical endocrinology and metabolism. 2004; 89: 2647-51.

49 Widdowson EM, McCance RA. The Effect of Finite Periods of Undernutrition at Different Ages on the Composition and Subsequent Development of the Rat. Proc R Soc Lond B Biol Sci. 1963; 158: 329-42.

50 Karaolis-Danckert N, Gunther AL, Kroke A, Hornberg C, Buyken AE. How early dietary factors modify the effect of rapid weight gain in infancy on subsequent bodycomposition development in term children whose birth weight was appropriate for gestational age. American Journal of Clinical Nutrition. 2007; 86: 1700-8.

51 Ong KK, Emmett PM, Noble S, Ness A, Dunger DB. Dietary energy intake at the age of 4 months predicts postnatal weight gain and childhood body mass index. Pediatrics. 2006; 117: e503-8.

52 Gluckman PD, Hanson MA, Cooper C, Thornburg KL. Effect of in utero and earlylife conditions on adult health and disease. N Engl J Med. 2008; 359: 61-73.

53 Griffiths L, Hawkins S, Cole T, Dezateux C. Risk factors for rapid weight gain in preschool children: Findings from a UK-wide prospective study. International Journal of Obesity. 2010; 34: 624-32.

This article is protected by copyright. All rights reserved. 
54 Oyama M, Nakamura K, Tsuchiya Y, Yamamoto M. Unhealthy maternal lifestyle leads to rapid infant weight gain: Prevention of future chronic diseases. Tohoku Journal of Experimental Medicine. 2009; 217: 67-72.

55 Lucas A. Growth and later health: a general perspective. Nestle Nutr Workshop Ser Pediatr Program. 2010; 65: 1-9; discussion 9-11.

56 Adair LS. Developing world perspective: the importance of growth for short-term health. Nestle Nutr Workshop Ser Pediatr Program. 2010; 65: 71-9; discussion 79-83.

\section{Figure legends}

Figure 1: Flow chart of study selection

Figure 2. Study-specific estimates and pooled odds ratio of overweight for those with RWG during early childhood compared to those without RWG.

Figure 3. Funnel plot of the studies which considered RWG as a predictor of overweight status.

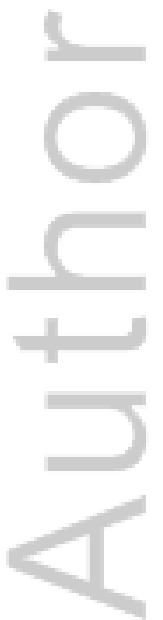

This article is protected by copyright. All rights reserved. 


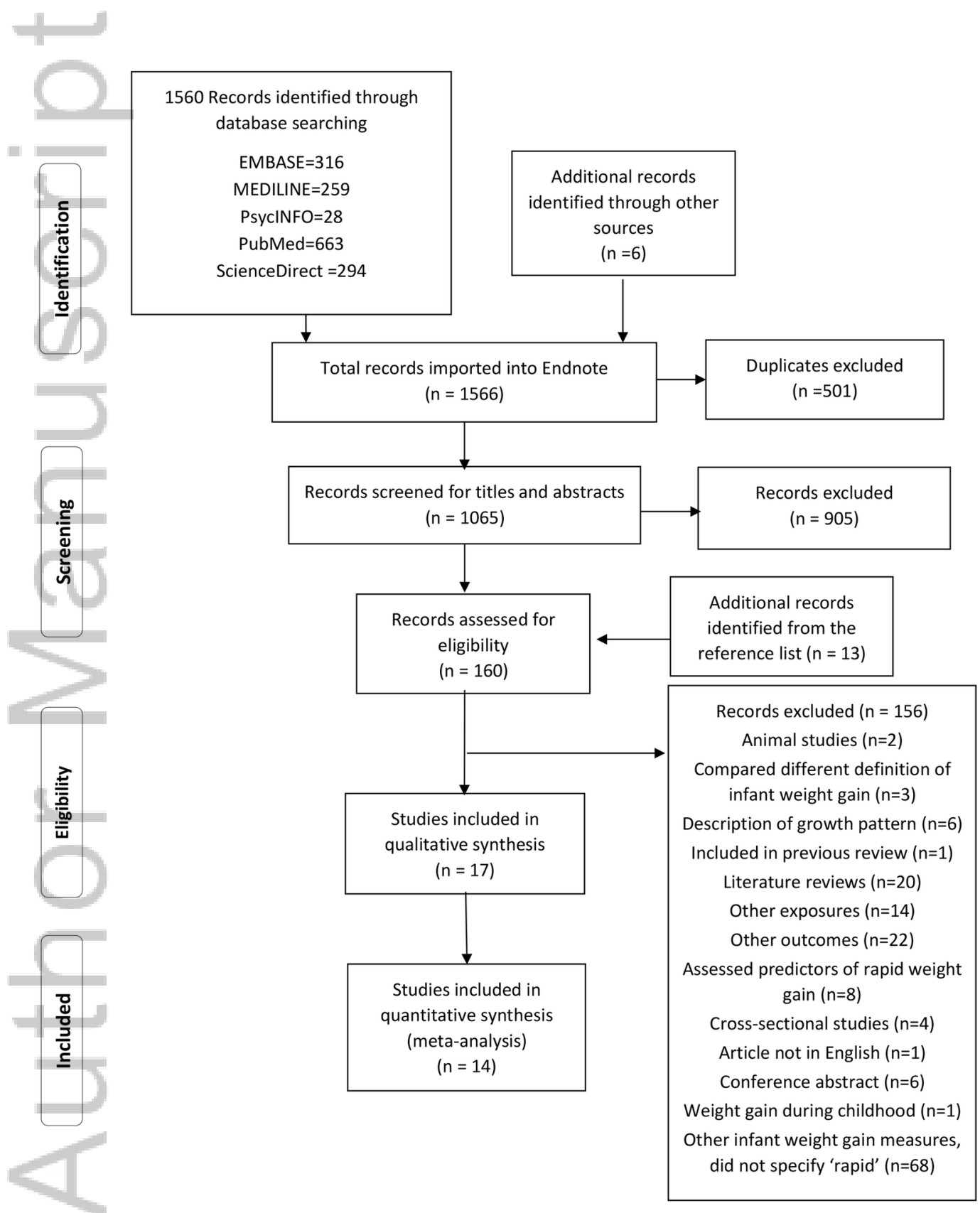

OBR_12632_F1.tif 


\section{Author (year)}

Karaolis-Danckert (2006)

Akaboshi (2008)- boys

Akaboshi (2008)- girls

Demerath (2009)

Goodell (2009)

Fujita (2013)

Odegaard (2013)

Rathnayake (2013)

Webster (2013)

Weng (2013)

Heppe (2013)

Goncalves (2014)

Sutharsan (2015)

Nanri (2016)- girls

Nanri (2016)- boys

Zhou (2016)

Overall

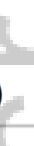

\section{$>$}

(5) 


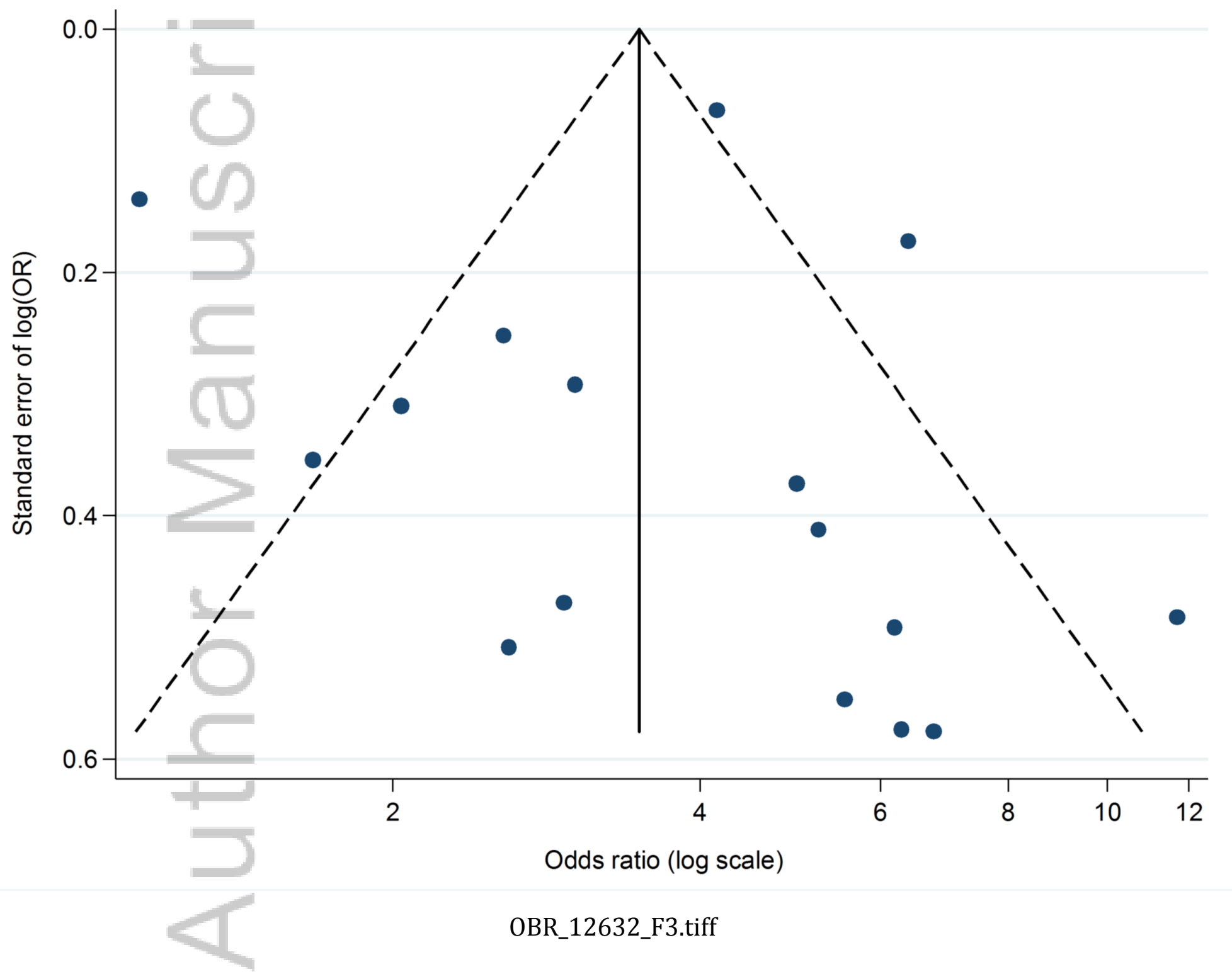

This article is protected by copyright. All rights reserved. 


\section{University Library}

\section{- M M N E R VA A gateway to Melbourne's research publications}

Minerva Access is the Institutional Repository of The University of Melbourne

Author/s:

Zheng, M;Lamb, KE;Grimes, C;Laws, R;Bolton, K;Ong, KK;Campbell, K

Title:

Rapid weight gain during infancy and subsequent adiposity: a systematic review and metaanalysis of evidence

Date:

2018-03-01

Citation:

Zheng, M., Lamb, K. E., Grimes, C., Laws, R., Bolton, K., Ong, K. K. \& Campbell, K. (2018). Rapid weight gain during infancy and subsequent adiposity: a systematic review and metaanalysis of evidence. OBESITY REVIEWS, 19 (3), pp.321-332. https://doi.org/10.1111/ obr.12632.

Persistent Link:

http://hdl.handle.net/11343/293749 